\title{
Oblique corpectomy in the cervical spine
}

\author{
Tomasz Tykocki $\mathbb{1}^{1} \cdot$ Łukasz A. Poniatowski $^{2,3} \cdot$ Marcin Czyz ${ }^{4} \cdot$ Guy Wynne-Jones ${ }^{1}$
}

Received: 5 May 2017 / Revised: 29 July 2017 / Accepted: 18 August 2017 / Published online: 5 December 2017

(C) International Spinal Cord Society 2018

\begin{abstract}
Study design A narrative review.

Objectives A literature review of studies reporting on the application of oblique corpectomy (OC) in various pathologies of the cervical spine.

Setting UK.

Methods A search was carried out using the PubMed and Google Scholar up to 18 March 2017. Finally, 26 studies met the inclusion criteria.

Results A multilevel OC shows good clinical outcomes in various pathologies in the cervical spine. The clinical improvement in cervical spondylotic myelopathy (CSM) and ossification of the posterior longitudinal ligament was found to be over 70\%. OC allows wide anterior decompression of the spinal cord and complete unilateral nerve root decompression. The approach carries a risk of Horner's syndrome, vertebral artery and accessory nerve injury. OC does not compromise spine stability and osteoarthrodesis with bone grafting is not necessary. Spinal motions are preserved and appear close to normal. OC can be applied in patients with a low fusion rate such as the elderly, diabetics, and heavy smokers. Furthermore, OC was found to be an optimal approach for exta-intradural tumors of the cervical spine.

Conclusions OC seems to be a valid alternative for the management of multisegmental CSM in selected cases. It should not be considered a first-line treatment strategy due to the relatively high morbidity. There are no studies comparing OC without fusion to other treatment options in CSM. Therefore, rigorous prospective studies using validated outcome measures with long-term follow-up are required.
\end{abstract}

\section{Introduction}

Cervical spondylosis is the most common cause of myelopathy in patients over 55 years of age and cervical spondylotic myelopathy (CSM) develops in almost all patients with $>30 \%$ narrowing of the cross-sectional area of the cervical spinal canal $[1,2]$. The natural course of symptoms is highly variable but generally in $~ 75 \%$ of cases with CSM, there is either slow or gradual progression [3-5]. In symptomatic patients, surgical decompression seems to be a procedure of choice. Surgical approaches, either anterior or

Tomasz Tykocki

ttykocki@gmail.com

Spinal Unit, Royal Victoria Infirmary, Newcastle, UK

2 Department of Experimental and Clinical Pharmacology, Medical University of Warsaw, Warsaw, Poland

3 Military Institute of Medicine, Warsaw, Poland

4 Royal Orthopaedic Hospital, Birmingham, UK posterior aim to widen the cervical canal and arrest the progression of myelopathy at first instance. The prognosis with surgery is worse with increasing severity of neurological deficits at the time of presentation and with longer duration of symptoms [6,7]. The oblique cervical corpectomy (OC) through a lateral cervical approach is an alternative to the central corpectomy. OC allows for multilevel decompression without the need for a bone graft or instrumentation, thus preserving motion during flexion and extension together with sagittal alignment. The main risks related to this procedure are vertebral artery (VA) and accessory nerve injury or Horner's syndrome [8-10].

The technique of $\mathrm{OC}$ has been clearly described by George et al. [10] (Fig. 1). Briefly, the patient is positioned supine with the head extended and rotated slightly to the contralateral side. The skin incision is along the anterior border of the sternocleidomastoid muscle, a dissection plane between the sternocleidomastoid muscle and the jugular vein is created, the neurovascular bundle is retracted medially to reach the cervical spine anterolaterally. Lee et al. [11] proposed a transverse skin incision using the 
Fig. 1 Schematic presentation of the anatomical approach in the oblique corpectomy. Dashed arrow shows the approach in the oblique corpectomy. $C C A$ common carotid artery, $I J V$ internal jugular vein, $S C M$ sternocleidomastoid muscle

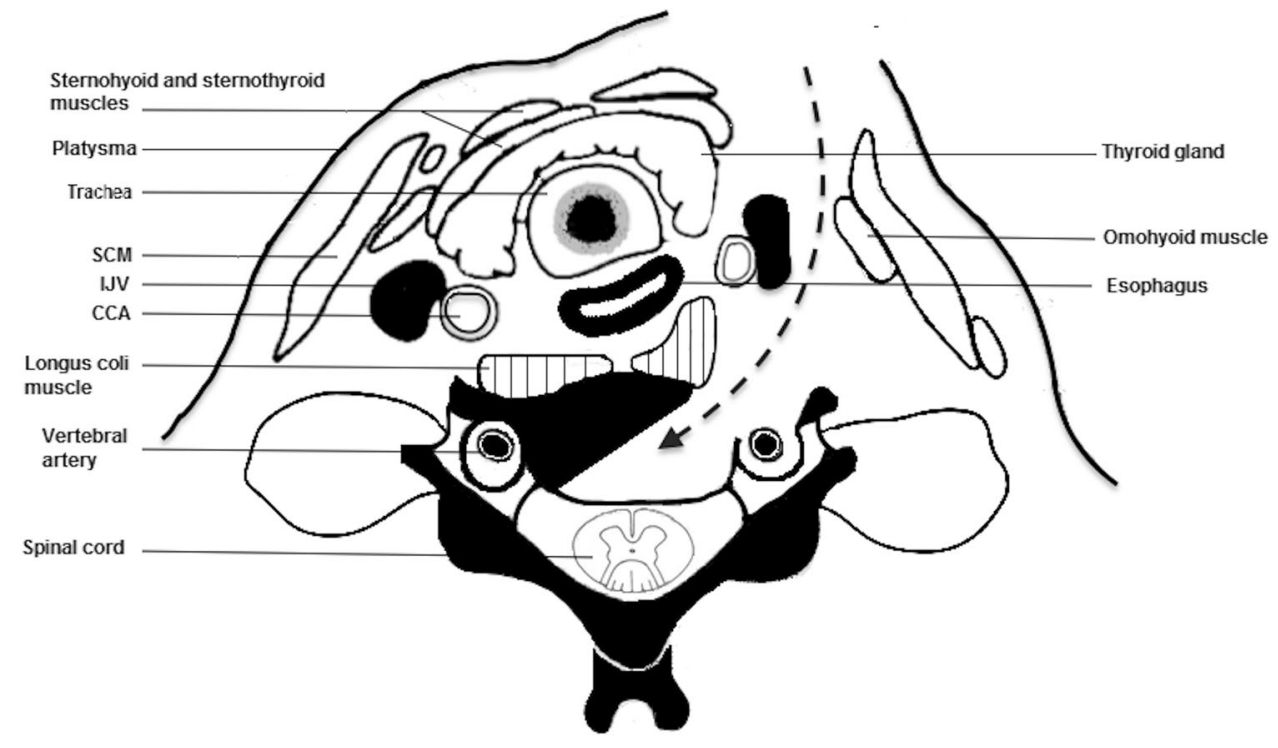

standard Smith-Robinson approach with distraction screws inserted in the center of the vertebral bodies as a guide to the midline and medial retractors. The longus capitis muscle is displaced laterally and the longus colli muscle, on which lies the sympathetic chain is retracted medially (laterally if a Smith-Robinson approach is used). A vertical drilling of the vertebral body should start from the lateral side, where the transverse process intersects with vertebral body, medial to VA. Drilling the costotransverse bars down to the periosteum allows to expose VA if necessary. Once the posterior longitudinal ligament is reached, further drilling is continued in an oblique trajectory to the contralateral side, at the last stage the PLL is opened.

The aim of this systematic review is to present up to date the data on the surgical application of the oblique corpectomy in various pathologies in the cervical spine. Authors predominantly focused on the postoperative clinical and radiological outcomes, biomechanical instability and complications related to $\mathrm{OC}$.

\section{Methods}

\section{Search strategy}

A search was carried out using the PubMed and Google Scholar up to March 18, 2017. Articles resulting from these searches and relevant references cited in those articles were reviewed. The search strategy used both keywords and the $\mathrm{MeSH}$ term searches for: cervical oblique corpectomy, corpectomies, vertebrotomy, which were combined with the appropriate Boolean connectors.

\section{Inclusion and exclusion criteria}

Clinical studies, biomechanical cadaveric studies and case reports on the application of OC for various cervical spine pathologies were included in the review. The selection process has been illustrated in Fig. 2. From 40 records found, three review studies, and three letters and comments were excluded. Four articles referring to the anterior cervical corpectomy and two others to lumbar or thoracic spine were removed. Two records were excluded due to the replicated data. Finally, 26 studies met the inclusion criteria. All studies were classified according to the evidence based medicine (Table 1).

\section{Results}

\section{Biomechanical studies}

The biomechanics and kinematic parameters of multilevel OC were first studied in vitro by Cagli et al. on human cadaveric spines (C3-Th1) [12]. A right-sided approach was performed at C5-6 and then tested using precise controlled loads in standard servohydraulic system associated with stereophotogrammetric three-dimensional angular motion analysis. An increase in motion was about one-third of that observed after standard unplated corpectomy with graft inducing significantly less instability and more motion in all directions. OC was associated with increased range of motion in all directions (15\% flexion, $18 \%$ extension, $11 \%$ lateral bending, $18 \%$ axial rotation) greater than normal with mean of $15^{\circ}$. Similar findings were reported by Karalar 
Fig. 2 Chart flow presenting study selection in the review

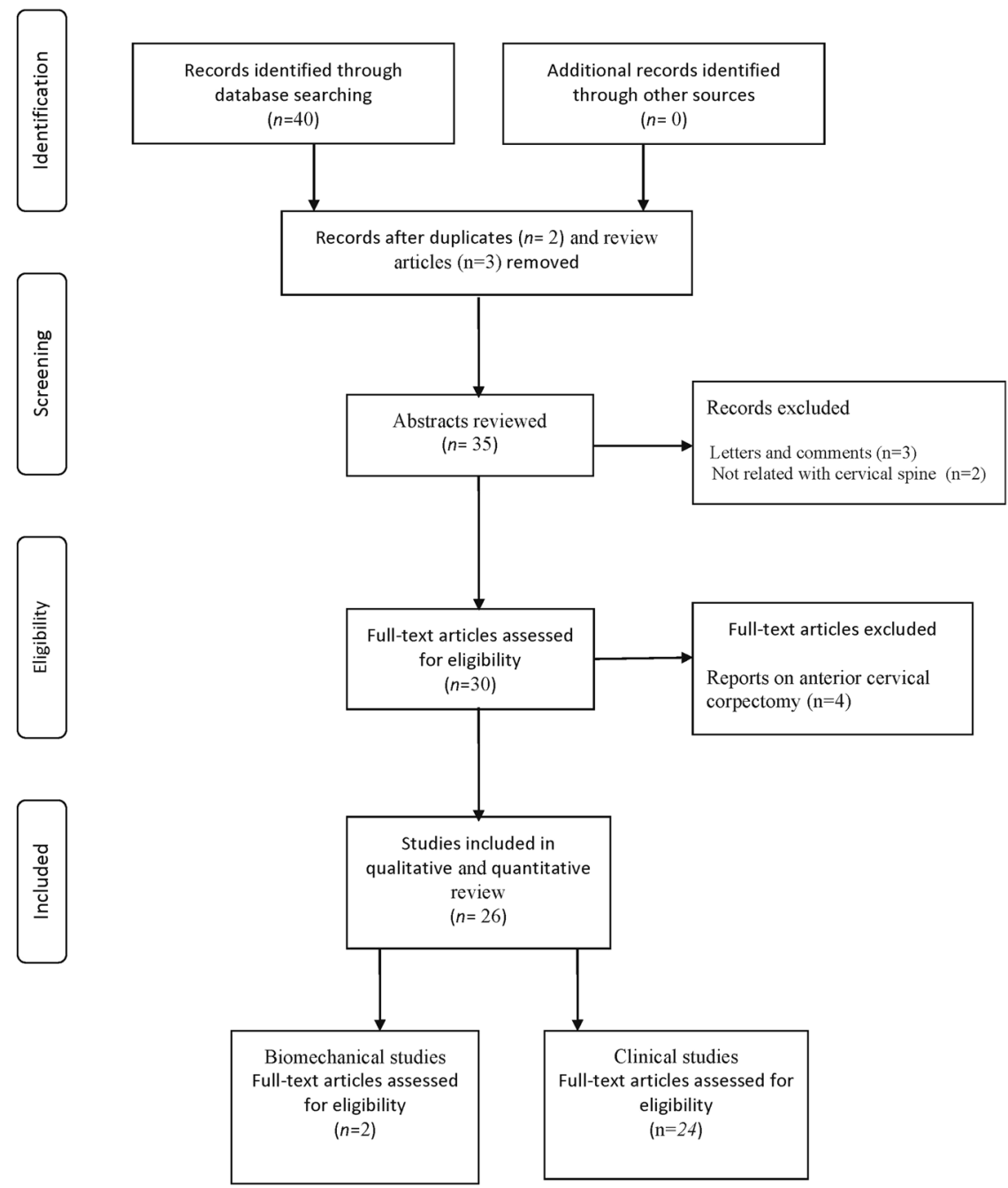

Table 1 Level of evidence

Level I Systematic review (with meta-analysis) of randomized clinical trials

Level II Cohort studies

Level III Case-control studies

Lelel IV Case series

Level V Expert opinion

et al. [13] on sheep models $(n=37)$. They also noted that the multilevel OC does not cause instability under physiological load deformation in flexion, extension, lateral bending, axial rotation, and axial loading.

In the study of Chacko et. al. [8], the amount of flexion and extension decreased significantly by a mean of $2.5^{\circ}$ and $7^{\circ}$, respectively. The residual range of movement across the operated segments for a single-level OC was $16.7^{\circ}(60 \%$ of the preoperative value), for two-level OC-20.0 $(67 \%)$ and for three-level $\mathrm{OC}-22.9^{\circ}(74 \%)$. Thirty precent of patients with preoperative lordosis developed a straight spine and 5 became kyphotic without clinical worsening. The incidence of loss of lordosis did not correlate with the number of operated levels. The six patients with preoperative kyphotic spines showed no change in whole spine curvature. None of the patients developed spinal instability.

\section{Cervical myelopathy and ossification of the posterior longitudinal ligament}

In 1999, George et al. [10] first proposed and described a new surgical technique called oblique corpectomy without interbody stabilization as an alternative procedure for the treatment of CSM and cervical spondylotic radiculopathy (CSR) [12, 14]. According to the authors, the main principles of OC through an anterolateral approach is a precise control of the VA and corpectomy preserving more than a half of the vertebral body, which could be performed on multilevels maintaining proper stabilization not requiring further fusion with graft and plate. The study group 
included total of 101 patients, 66 with CSM and 35 CSR. The multilevel OC without interbody stabilization was performed on one to five levels from $\mathrm{C} 2-\mathrm{C} 3$ to $\mathrm{C} 7-\mathrm{Th} 1$. The oblique corpectomy was performed in patients with anterior cervical compression, with reduced lordosis and without instability of the cervical spine. Instability was defined as the presence of preoperative anterolisthesis $>2 \mathrm{~mm}$ on dynamic X-ray. Neither the number of vertebral levels involved in the spondylotic process nor the presence of congenital canal stenosis influenced the selection process. Authors included patients with anterior spinal cord compression and loss of physiological lordosis of the cervical spine axis. The clinical improvement at mean follow-up of 37 months (range 2-66 months) in Japanese Orthopaedic Association (JOA) and Nurick grade (NG) scales was found in 82 and $67 \%$ of patients respectively, worsening in $8 \%$ in both scales.

The largest analysis of the OC on 499 patients with CSM and CSR was reported by Chibbaro et al. [9]. Authors included patients with clinical evidence of cervical myelopathy and/or radiculopathy, single/multiple level anterolateral nerve roots and/or spinal cord compression, neutral or kyphotic cervical alignment without instability on plain/ dynamic radiographs. Patients improvement was evaluated using a global recovery formula: (postop JOA score preop mJOA score) $\times 100 / 17$ - preop JOA score. A global recovery rate of $88 \%$ was found for CSM and $95 \%$ for CSR at mean follow-up of 111 months (range: 9-202 months), A total of 900 levels were decompressed predominantly at C4-C5 (188/21\%), C5-C6 (296/33\%), C6-C7 in (128/ 14.2\%). 221 (24.6\%) patients had one level and 121 (13.4\%) two levels decompressed, in $9(1 \%)$ cases a fivelevel OC was performed. In three patients delayed stabilization was required due to a disc herniation, an unrecognized congenital bone malformation and segmental instability at a level above the surgery.

In a 14-year prospective study on OC in CSM Chibbaro et al. [15] analyzed 268 patients treated by multilevel OC technique without bone grafting and additional instrumentation. They included patients with clinical evidence of cervical myelopathy, sphincter disturbances not explained otherwise, multilevel anterior cervical compression on MRI and neutral/kyphotic cervical alignment without instability on plane radiographs. The multilevel OC without instrumented stabilization was performed on one to five levels from C3 to Th1 with total decompression of 527 levels. Clinical improvement in JOA score occurred in $87 \%$ of the patients during average 96 months period of follow-up, worsening in 5\% and no changes were observed in $8 \%$. A significant improvement in Neck Disability Index score was seen at 6 weeks after surgery (55.2 vs. 31.2). In another prospective study on 13 CSM and 13 CSR patients [16], a clinical improvement in JOA score was observed in $77 \%$ of
CSM cases and no change in $23 \%$. Good outcome in CSR group was in $85 \%$ of patients and $8 \%$ had persistent pain. Postoperative radiological results showed that the mean Pavlov ratio (the ratio between the spinal canal and the VB diameter) increased by $76.2 \%$ from 0.72 to 1.26 and sagittal cervical canal diameter improved an average of $67 \%$.

Very good results were presented by Kiris et al. [17] in the prospective study on 40 patients with CSM and 8 with additional radicular symptoms. The clinical improvement in JOA score was observed in $92.5 \%$ of patients at 6 months of follow-up, worsening in $2.5 \%$ and no changes in $5 \%$. The mean preoperative JOA score of $12.8 \pm 3.1$ significantly changed to $14.9 \pm 2.6$ postoperatively. This study found a positive correlation between preoperative JOA score and preoperative spinal canal diameter, which also negatively correlated with the time of surgery. In the study of Rocchi et al. [18] on 48 patients, the mean improvement at 2-year follow-up in both JOA and NG occurred in $85 \%$ of cases, worsening in $10 \%$ and no changes were observed in $4 \%$. The total functional recovery (JOA score of 16 or 17) was achieved in 45\% (22 out of 48) and all nine patients with CSR were pain free. All patients showed spinal stability.

In two retrospective studies by Chacko et al. [8, 19], a total of 109 and 153 patients were analyzed, respectively. Sixteen cases with ossification of the posterior longitudinal ligament (OPLL) were also included. Majority of patients presented with spastic gait $(95 \%)$, paresthesia $(82 \%)$ and impaired proprioception (6\%). $83 \%$ underwent, either a single- or two-level OC. Men constituted $93 \%$ of all cases. Clinical outcomes showed significant improvement in NG by $30 \%$ and in JOA by $24 \%$. Immediate postoperative improvement in spasticity and paraesthesia was seen in $73 \%$ patients, one fourth remained the same while five cases worsened due to a C5 radiculopathy. All $13 \mathrm{NG} \mathrm{V}$ patients improved to a better grade. Of these, $46 \%$ improved to Grade I or II and 54\% to Grade III or IV.

The same authors found that the whole spine (Cobb angles between the lower endplate of $\mathrm{C} 2$ and the upper endplate of $\mathrm{C} 7$ on lateral cervical radiographs in flexion and extension) and segmental range of motion decreased significantly by $11.2^{\circ}$ and $10.9^{\circ}$, respectively at mean followup of 45 months [20]. There was an extensive osteophyte progression and ossification of the anterior longitudinal ligament in two-thirds of cases (increase in Nathan's grade), more pronounced at the level of surgery, but also at the adjacent segments cranially and caudally in about half of cases.

In the prospective study on 24 patients with CSM and OPLL Moses at al. [21] investigated the utility of intraoperative ultrasound (IU). Although 20 out of 24 (83\%) patients achieved a clinical improvement in NG, results did not confirm a significant correlation between immediate reexpansion of the spinal cord after the decompression and 
the clinical improvement in patients with both, OPLL or CSM. The negative predictive value of neurological improvement with a cord expansion of $>1 \mathrm{~mm}$ was $14 \%$ ( 2 out of 14). IU identified the VA between the transverse processes of the levels exposed in all cases. However, in two cases with OPLL an adequate imaging was impossible due to shadowing from calcific residues in the dura. In six cases, IU found residual compression of the dura and the cord on the contralateral side of the canal after the surgeon's opinion of complete corpectomy. In two patients, IU failed to detect residual compression that were seen on the postoperative CT scans. Authors compared incidence of residual bony compression in cases operated with and without IU, but did not find any difference. Measurement of the width of the OC by IU proved to be accurate as compared with postoperative computed tomography (CT) and magnetic resonance imaging (MRI) measurements.

Lee et al. [11] performed OC in 22 patients with OPLL. In half of the cases, a $\mathrm{C}$-arm-based intraoperative navigation was used, and the remaining 11 were operated on without navigation. They used a standard Smith-Robinson approach for OC. The improvement in JOA score at 1 year was $59.6 \%$ (5.6 points) in the navigation group and $35.2 \%$ (3.3 points) in non-navigation group. The application of image guided navigation allowed to achieve more complete OC and to preserve the entire anterior portion of the upper and/ or lower end of decompression through undermining drilling on sagittal oblique plane.

Goel et al. [22] presented four cases with OPLL and myelopathy treated with multilevel OC technique [17]. During the follow-up that ranged from 6 months to 5 years, all patients showed improvement in neurological symptoms with satisfactory anatomical decompression. There was no instability in any of cases. Another report on three patients with OPLL and a coexisting ossified anterior longitudinal ligament (OALL) showed a clinical and radiological improvement at 6-, 12- and 36-month follow-up period [14]. Authors concluded that multiple OC technique could be valuable therapeutic option for patients with OALL in case of progressive myelopathy due to OPLL with intrinsic stability provided by presence of OALL.

\section{Complications}

The most common postoperative complication after OC was temporary Horner's syndrome (3-57\%) and permanent in $0.4-8 \%[8,10,15,18,19]$. The cumulative rate of transient Horner's syndrome was $15.7 \%$ (73 out of 465 ) and permanent $3.4 \%$ (16 out of 465 ). However a modification of the operative technique by making a longitudinal incision in the longus colli sheath and retracting the sympathetic chain medially was proposed to reduce the incidence of this complication [8].
There was one report on the VA injury during dissection of the longus colli [8]. Additional complications were axial pain $(8 \%)$, kyphosis/axial pain (4\%), persistent brachialgia (4\%), cerebrospinal fluid fistula $(4 \%)$, contralateral foraminal stenosis $(4 \%)$, hematoma $(2.5 \%)$, nerve root injury and perforation of the lymphatic duct in one case $[8,10,15$, 17]. Five patients developed a $\mathrm{C} 5$ radiculopathy in the immediate postoperative period, four of which improved to normal in 6 months [8]. Cervical spine instability was reported in 3 cases [10].

Patients with OPLL had significantly higher rate of postoperative complications, including CSF leak, radicular deficit, lower range of movement and lower NG at last follow-up [19].

\section{Radiological studies}

Sarkar at al. [23] presented radiological and clinical results of OC in 56 myelopathic patients. Authors investigated T2weighted intramedullary increased signal intensity (ISI) on pre- and postoperative MRI, and also analyzed clinical status in NG and JOA scales. The characteristics of the ISI quality was based on the following system: Type 0 none, Type 1-fuzzy, Type 2-sharp, and Type 3-mixed. Authors postulate that reversible cord edema and ischemia appear as fuzzy changes and cavitation and necrosis as sharp lesions on T2-weighted MRI. Most preoperative ISI changes were Type $1(41 \%)$ and $3(34 \%)$. In more than three-quarters of patients with type 3 lesions the postoperative ISI change was improved to type 2 (sharp and focal). This means that surgical decompression reverses the extensive cord edema with diffuse changes on MRI, leaving behind small residual focal, necrotic and cavitatory lesions. Multisegmental and Type 3 lesions showed a significant propensity to regress after surgery reducing to Type 2 changes at follow-up. Type 2 of ISI, either on preoperative or postoperative imaging, may negatively impact outcomes, but these associations were not statistically significant. A regression of the ISI by $>50 \%$ at 18 months of follow-up predicts better functional outcomes.

Turel at al. [24] presented two patients with an occurrence of extensive T2-weighted changes and contrast enhancement on T1 MRI extending far beyond the levels of OC with a significant improvement of neurological deficits postoperatively. However, in both cases, a follow-up MRI at 2 years showed resolution of the radiological changes and no instability albeit with a mild kyphosis on dynamic cervical X-rays showed. Rapid decompression of the hypoperfused spinal cord results in an hyperaemic and 'extra perfusion' state with poor initial autoregulation at the compression level [25]. The radiological appearance of this cascade is a hyperintensity on T2-weighted imaging corresponding to spinal cord edema. 


\section{Tumors, infection and vascular pathologies}

Kunert et al. [26] treated four patients with a 2-level OC for spinal epidural abscess. All patients presented with progressive myelopathy. Follow-ups ranged from 3 days to 9.2 years (mean: 5.5 years). One patient fully recovered immediately after the surgery and two others presented with significant neurological improvement. One, 90-year-old patient died on the third postop day due to cardiac arrest. A postop course did not show a recurrent abscess and no instrumental stabilization was necessary for any of the cases. The same authors used OC for left VA dissection with intramural hematoma who presented with left radiculopathy at C5 and C6 [27]. The radicular pain resolved immediately and sensorimotor deficit completely disappeared within 4 months. Imaging of VA at 6 months postoperative showed normal picture of the vessel without residual hematoma. There were no ischemic events within 2,5 years of follow-up.

Radek at al. [28] performed OC in 33-year-old woman with ventral meningioma at $\mathrm{C} 5-\mathrm{C} 6$. This patient presented with spastic paraparetic gait, weak tendon reflexes in the upper extremities, exaggerated knee jerk reflexes and bilateral Babinski sign. They used left-sided approach and achieved a total tumor resection. Due to an extensive and medial vertebral bodies resection, authors decided to perform spinal fusion of C4-C7 with right iliac crest graft and titanium plate and five screws were used for stabilization. OC was also performed in 14-year-old girl with cervical chordoma who presented with cervical pain, dysphagia, hyperreflexia and weakness of the four limbs for 8 months [29]. The preoperative MRI showed a lesion of $\mathrm{C} 2-\mathrm{C} 4$ with marked retropharyngeal and epidural space involvement. A total tumor removal was achieved by a precarotid and retrocarotid approach during a single operation. A C2-C4 discectomy and $\mathrm{OC}$ with anterior plating was performed. The patient was free of any neurological deficit on the second day postoperation. She received postoperative proton beam radiation therapy and no recurrence or metastasis was observed 48 months after the operation.

Fontaine et al. [30] performed an anterolateral approach combined with multiple OC technique in a case of anterior intramedullary cavernous angioma with an exophytic extramedullary portion. A 42-year-old woman presented with gradually worsened neurological condition upon time, leading to a severe tetraplegia predominating in the lower limbs and hypoesthesia of all four limbs. To avoid myelotomy, an $\mathrm{OC}$ was performed at $\mathrm{C} 4$ followed by $\mathrm{C} 3-\mathrm{C} 4$ and $\mathrm{C} 4-\mathrm{C} 5$ discectomies with preservation of half of the vertebral body and anterior longitudinal ligament. OC provided a direct access to the pathological lesion allowing its excision, after then, bone graft was collected from the iliac crest and inserted between $\mathrm{C} 3$ and $\mathrm{C} 5$, which was stabilized by plate. A deterioration of motor functions without impairment of sensory function was observed postoperatively. At 6 months postoperation, upper limb weakness had improved, but paraparesis persisted, sphincter disturbances had relieved. Nishikawa et al. [31] also reported three cases with ventral cervical intramedullary cavernous angiomas approached via $\mathrm{OC}$ at $\mathrm{C} 2$ and $\mathrm{C} 3$ levels. All patients presented with numbness in the upper extremities. Authors performed a standard Smith-Robinson approach to reach the prevertebral space. Iliac bone grafts were used in all patients and additionally locking screws and plates in two cases. Postoperatively, all three patients improved neurologically and there was no postoperative morbidity.

OC was also applied in a 71-year-old man with an intradural-extramedullary schwannoma ventral to the spinal cord at the C3 level who presented with neck pain and dysesthesia in his right arm and both legs [32]. Patient also reported walking fatigability and neck pain during right lateral cervical flexion. The tumor was easily found in the dural sac and was totally removed. The symptoms improved after the operation. There was no instability on follow-up dynamic $\mathrm{x}$-ray at 12 months.

Lot et al. [33] presented a series of 57 patients with cervical neuromas, in five cases of tumors, OC through the anterolateral approach was performed [11]. OC was found to be an optimal approach allowing the exposure of dumbbell tumors with extra and intradural extensions with good control of the proximal and distal part of the cervical nerve root. There was no need for an extensive drilling of the vertebral body beyond the midline; additionally, an opening of intervertebral foramen provided a wide access to the anterolateral aspect of the dural sac. Authors made a longitudinal incision of the dura starting from the root exit zone and directed upward and downward.

Clinical data and literature summary was presented in Table 2.

\section{Conclusions}

A multilevel oblique corpectomy through an anterolateral approach shows good clinical outcomes in various pathologies of the cervical spine. However, this is rather a demanding technique that carries risk of VA injury together with a high rate of Horner's syndrome. Therefore, adequate knowledge of VA variations is essential and preoperative analysis of the anatomy of VA is mandatory. Furthermore, OC was found to be an optimal approach for exta-intradural tumors of the cervical spine, especially for those located in the ventral part of the spinal canal. The main advantage attributed to this technique is the adequate ventral decompression without the need for bony fusion and stabilizastion. 


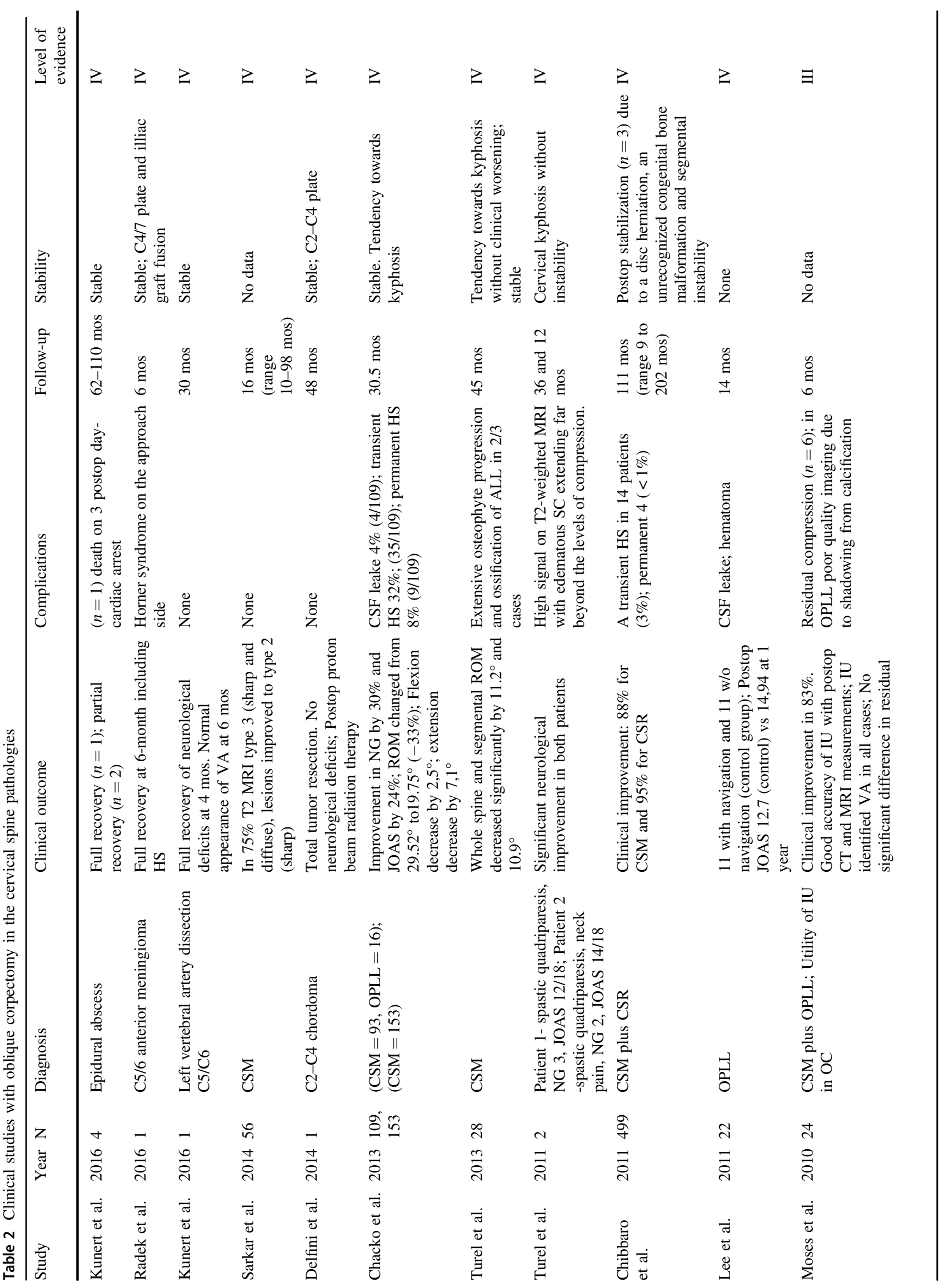




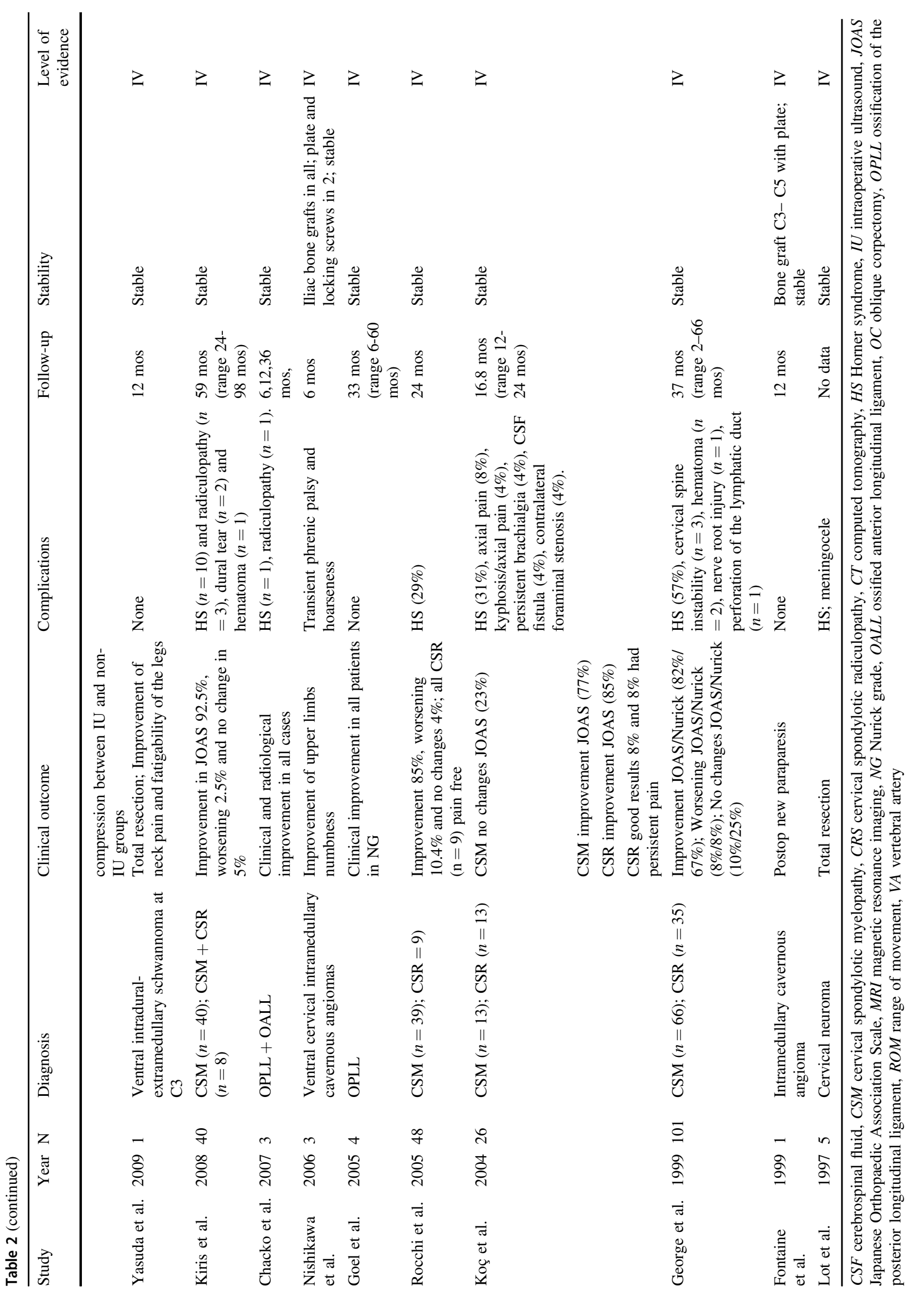


OC can be taken into account in the management of multisegmental cervical spondylosis and OPLL in selected cases. It should not be considered a first-line treatment strategy due to the relatively high morbidity associated with this procedure. The main concern is a relatively high rate of postoperative Horner's syndrome sharply contrasting with other approaches.

The clinical improvement in CSM, CRS and OPLL patietns was found to be at least $70 \%$ or more, which is similar to what is observed in studies with the central corpectomy. This technique does not compromise stability and bone grafting is not necessary, allowing the application of $\mathrm{OC}$ in patients with a low fusion rate such as the elderly, diabetics, and heavy smokers.

There are no studies comparing oblique corpectomy without fusion to other treatment options in CSM. Therefore, rigorous prospective studies using validated outcome measures with long-term follow-up are required.

Conflict of interest The authors declare that they have no conflict of interest.

\section{References}

1. Houser OW, Onofrio BM, Miller GM, Folger WN, Smith PL. Cervical spondylotic stenosis and myelopathy: evaluation with computed tomographic myelography. Mayo Clin Proc. 1994:69:557-63.

2. Yu YL, du Boulay GH, Stevens JM, Kendall BE. Computed tomography in cervical spondylotic myelopathy and radiculopathy: visualisation of structures, myelographic comparison, cord measurements and clinical utility. Neuroradiology. 1986;28:221-36.

3. Karadimas SK, Erwin WM, Ely CG, Dettori JR, Fehlings MG. Pathophysiology and natural history of cervical spondylotic myelopathy. Spine. 2013;38:S21-36.

4. Lees F, Turner JW. Natural history and prognosis of cervical spondylosis. Br Med J. 1963;2:1607-10.

5. Nurick S. The natural history and the results of surgical treatment of the spinal cord disorder associated with cervical spondylosis. Brain J Neurol. 1972;95:101-8.

6. Edwards CC, Riew KD, Anderson PA, Hilibrand AS, Vaccaro AF. Cervical myelopathy. current diagnostic and treatment strategies. Spine J. 2003;3:68-81.

7. Matz PG, Holly LT, Mummaneni PV, Anderson PA, Groff MW, Heary RF, et al. Anterior cervical surgery for the treatment of cervical degenerative myelopathy. J Neurosurg Spine. 2009;11:170-3.

8. Chacko AG, Turel MK, Sarkar S, Prabhu K, Daniel RT. Clinical and radiological outcomes in 153 patients undergoing oblique corpectomy for cervical spondylotic myelopathy. Br J Neurosurg. 2014;28:49-55.

9. Chibbaro S, Orphee M, Damien B, Alisha R, Pavel P, Bernard G. Oblique corpectomy to manage cervical myeloradiculopathy. Neurol Res Int. 2011;2011:734232.

10. George B, Gauthier N, Lot G. Multisegmental cervical spondylotic myelopathy and radiculopathy treated by multilevel oblique corpectomies without fusion. Neurosurgery. 1999;44:81-90.

11. Lee H-Y, Lee S-H, Son HK, Na JH, Lee JH, Baek OK, et al. Comparison of multilevel oblique corpectomy with and without image guided navigation for multi-segmental cervical spondylotic myelopathy. Comput Aided Surg. 2011;16:32-37.

12. Cagli S, Chamberlain RH, Sonntag VKH, Crawford NR. The biomechanical effects of cervical multilevel oblique corpectomy. Spine. 2004;29:1420-7.

13. Karalar T, Unal F, Güzey FK, Kiris T, Bozdag E, Sünbüloglu E. Biomechanical analysis of cervical multilevel oblique corpectomy: an in vitro study in sheep. Acta Neurochir (Wien). 2004;146:813-8.

14. Chacko AG, Daniel RT. Multilevel cervical oblique corpectomy in the treatment of ossified posterior longitudinal ligament in the presence of ossified anterior longitudinal ligament. Spine. 2007;32:E575-80.

15. Chibbaro S, Mirone G, Makiese O, George B. Multilevel oblique corpectomy without fusion in managing cervical myelopathy: long-term outcome and stability evaluation in 268 patients. J Neurosurg Spine. 2009;10:458-65.

16. Koç RK, Menkü A, Akdemir H, Tucer B, Kurtsoy A, Oktem IS. Cervical spondylotic myelopathy and radiculopathy treated by oblique corpectomies without fusion. Neurosurg Rev. 2004;27:252-8.

17. Kiris T, Kilinçer C. Cervical spondylotic myelopathy treated by oblique corpectomy: a prospective study. Neurosurgery. 2008;62:674-682.

18. Rocchi G, Caroli E, Salvati M, Delfini R. Multilevel oblique corpectomy without fusion: our experience in 48 patients. Spine. 2005;30:1963-9.

19. Chacko AG, Joseph M, Turel MK, Prabhu K, Daniel RT, Jacob KS. Multilevel oblique corpectomy for cervical spondylotic myelopathy preserves segmental motion. Eur Spine J. 2012;21:1360-7.

20. Turel MK, Sarkar S, Prabhu K, Daniel RT, Jacob KS, Chacko AG. Reduction in range of cervical motion on serial long-term follow-up in patients undergoing oblique corpectomy for cervical spondylotic myelopathy. Eur Spine J. 2013;22:1509-16.

21. Moses V, Daniel RT, Chacko AG. The value of intraoperative ultrasound in oblique corpectomy for cervical spondylotic myelopathy and ossified posterior longitudinal ligament. Br J Neurosurg. 2010;24:518-25.

22. Goel A, Pareikh S. Limited oblique corpectomy for treatment of ossified posterior longitudinal ligament. Neurol India. 2005;53:280-2.

23. Sarkar S, Turel MK, Jacob KS, Chacko AG. The evolution of T2weighted intramedullary signal changes following ventral decompressive surgery for cervical spondylotic myelopathy: Clinical article. J Neurosurg Spine. 2014;21:538-46.

24. Turel MK, Chacko AG. Delayed resolution of extensive T2weighted intramedullary signal changes after oblique corpectomy for cervical spondylotic myelopathy. $\mathrm{Br} \mathrm{J}$ Neurosurg. 2011;25:772-4.

25. Lee S-W, Antiga L, Steinman DA. Correlations among indicators of disturbed flow at the normal carotid bifurcation. J Biomech Eng. 2009;131:61013.

26. Kunert P, Prokopienko M, Nowak A, Czernicki T, Marchel A. Oblique corpectomy for treatment of cervical spine epidural abscesses: report on four cases. Neurol Neurochir Pol. 2016;50:491-6.

27. Kunert P, Prokopienko M, Czernicki T, Nowak A, Marchel A. Sensorimotor C5 and C6 radiculopathy caused by thrombosed vertebral artery dissection and successfully treated with limited oblique corpectomy - Case report. Neurol Neurochir Pol. 2016;50:48-51.

28. Radek M, Grochal M, Tomasik B, Radek A. The antero-lateral approach with corpectomy in the management of the ventral meningioma of the spinal canal. Neurol Neurochir Pol. 2016;50:226-30. 
29. Delfini R, Marruzzo D, Tarantino R, Marotta N, Landi A. Multilevel oblique corpectomies as an effective surgical option to treat cervical chordoma in a young girl. World $\mathrm{J}$ Clin Cases WJCC. 2014;2:57-61.

30. Fontaine D, Lot G, George B. Intramedullary cavernous angioma. Resection by oblique corpectomy. Surg Neurol. 1999;51: 435-41.

31. Nishikawa M, Ohata K, Ishibashi K, Takami T, Goto T, Hara M, The anterolateral partial vertebrectomy approach for ventrally located cervical intramedullary cavernous angiomas. Neurosurgery. 2006;59:ONS58-63

32. Yasuda M, Bresson D, Cornelius JF, George B, Anterolateral approach without fixation for resection of an intradural schwannoma of the cervical spinal canal: technical note. Neurosurgery. 2009;65:1178-81

33. Lot G, George B. Cervical neuromas with extradural components: surgical management in a series of 57 patients. Neurosurgery. 1997;41:813-20. 